\title{
Lesion Detection and Classification techniques for Diabetic Retinopathy
}

\author{
Jyoti P. Sawant, Sachin A. Naik, Santosh S. Chowhan
}

\begin{abstract}
Diabetes is a worldwide spread disease which is increasing rapidly and found in all age people. Diabetic Retinopathy is a retinal abnormality caused by diabetes. Which can lead to permanent vision loss or blindness. As Diabetic Retinopathy pathology damages retina without any early symptoms, it is very important to do the regular screening of retina and detection of Retinopathy. Ophthalmologist does the identification of Retinopathy manually which is time consuming and error prone. Hence, there is a need for early and correct automatic detection of Diabetic Retinopathy. Many researches have done for detection using Image Processing, Artificial Intelligence, Neural Network and Machine Learning. This paper presents a review on Diabetic Retinopathy Detection systems. This review highlights the public datasets available for the evaluation of the detection systems with different segmentation and classification techniques. We have discussed the analysis of different classification and segmentation techniques used in DR detection.
\end{abstract}

Keywords: Classification, Diabetic Retinopathy, Diabetic Retinopathy dataset, DR Lesions, DR Lesion Segmentation, Feature Extraction.

\section{INTRODUCTION}

Diabetic is a disease found worldwide in both developing and developed countries. As per reports in 2017, 425 million cases of diabetes with both Type-1, Type-2 and other types. As per estimates in 2013 showing 382 million people with diabetes, actual figure is very high. It may increase globally by $48 \%$ in year 2045. Diabetic Retinopathy is a retinal abnormality caused by damage of retinal blood vessels due to diabetes. Diabetic Retinopathy can lead to the vision loss. As, its early symptoms are not seen there is high risk of blindness in diabetic patients. Regular and early eye screening is suggested by the doctor to avoid vision loss. All Type- 1 diabetic patients and more than 60\% Type-2 diabetic patients suffer with Diabetic Retinopathy. So, early as well as accurate screening and detection of Retinopathy lesions is important to resist from blindness. Fundoscopic images are mostly used for detection, analysis and diagnosis of lesions in Diabetic Retinopathy. For the accuracy of results scientists are developing different Computer Aided Diagnosis (CAD) techniques. In contrary, manual screening is time consuming and has low detection accuracy.

\section{Revised Manuscript Received on February 05, 2020.}

* Correspondence Author

Jyoti P. Sawant*, Research Scholar, Faculty of Computer Studies, Symbiosis International University (Deemed University), Pune, Maharashtra, India. Email: Jyoti.sawant13@gmail.com.

Sachin A. Naik, Asst. Professor, Symbiosis Institute of Computer Studies and Research, Symbiosis International University (Deemed University), Pune, Maharashtra, India Email: Sachin.naik@sicsr.ac.in.

Santosh S. Chowhan, Asst. Professor, Symbiosis Institute of Compute Studies and Research, Symbiosis International University (Deemed University), Pune, Maharashtra, India. Email: santosh.chowhan@sicsr.ac.in.

(C) The Authors. Published by Blue Eyes Intelligence Engineering and Sciences Publication (BEIESP). This is an open access article under the CC BY-NC-ND license (http://creativecommons.org/licenses/by-nc-nd/4.0/)
Many researches have done on implementation of algorithms and Diabetic Retinopathy techniques for analysis of fundus, OCT and angiographic retinal images. These algorithms can efficiently and accurately does feature extraction, segmentation and classification of DR lesions and abnormalities. This paper presents analysis of existing Diabetic Retinopathy detection algorithms and techniques developed from 2008 to 2019.

The fundus image in "Fig. 1" shows the retinal image with pathologies of Diabetic Retinopathy. Which shows different lesions like microaneurysm, Hemorrhages, cotton wool spots, exudates and abnormal growth of blood vessels. In this paper we are presenting review on different automatic DR detection systems having few or all steps of feature extraction, segmentation, detection and classification techniques.

This paper describes the overview of Diabetic Retinopathy detection systems including lesion detection, segmentation and DR classification. Information of public datasets, available for the retinal analysis is provided. In this paper we have enlisted feature extraction techniques, segmentation techniques, classification techniques and their evaluated accuracy which provides the further research dimension in the field of diabetic retinopathy.

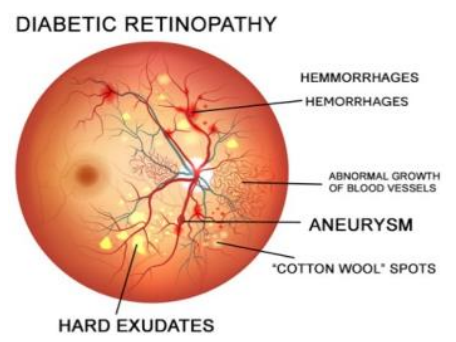

Fig. 1. Pathology of Diabetic Retinopathy

\section{OVERVIEW OF DIABETIC RETINOPATHY DETECTION SYSTEMS}

\section{A. Types of DR Detection Systems}

As observed in literature review Diabetic Retinopathy research can be categories depends upon type of study as follows:

1) Retina:

In this study all retinal factors are identified, segmented and classified. All DR lesions are identified. Focus is on full retina, so image analysis is done on full retina in [4], [5], [6], [7], [9], [10], [11], [12], [13]. 
Reference [1] proposed an automatic framework which classify and segment BRAO (Branch retinal artery occlusion) based on 3D Spectral-Domain Optical Coherence Tomography (SD-OCT) images. SVM Classifier is used for classification. A segmentation algorithm which detects optic disc and retinal exudates is proposed in [3]. Algorithm is compared with human graders on robustness and accuracy [3]. DR image classification using SVM and KNN classifiers is proposed in [19]. Images are analyzed with discrete wavelet transform.

2) Blood vessels:

This type of studies identifies and segments only blood vessels or vasculature of retina. It also identifies the early stage DR lesions related to blood vessels and abnormal growth of the blood vessels [11], [14], [15], [16], [17], [18], [20]. Authors have proposed a blood vessel segmentation method which has used Hessian matrix in [2]. Mathematical morphology is used for blood vessel segmentation by author in [8]. K. S. Argade et. al have combined data mining and image processing techniques for DR detection in [15].

3) Fundus Images:

DR studies can be categories on the basis of type of acquisition and retinal images. These types are Fundus images [3], [4], [7], [9], [17], [19], [20], fluorescein angiography, 2D, and 3D images. The proposed system is tested on color fundus images in [3].

"Fig. 2" shows categories of DR research as per literature review.

\section{B. Classification of DR Detection Systems Based on Detection of Abnormalities in Retina}

DR detection systems are classified by considering type of lesions they detect. The lesions considered for classification are OD (Optical Disk), retinal layer, blood vessel, macula, exudates, hemorrhage and microaneurysms. "Fig. 3", shows the classification of DR detection systems by its lesion detection type. We found that these seven types of lesions are detected to identify the DR stages and its severity. "Fig. 4.", describes the articles presented in literature as per the lesions. We observed that less work is done on multiple lesion detection.

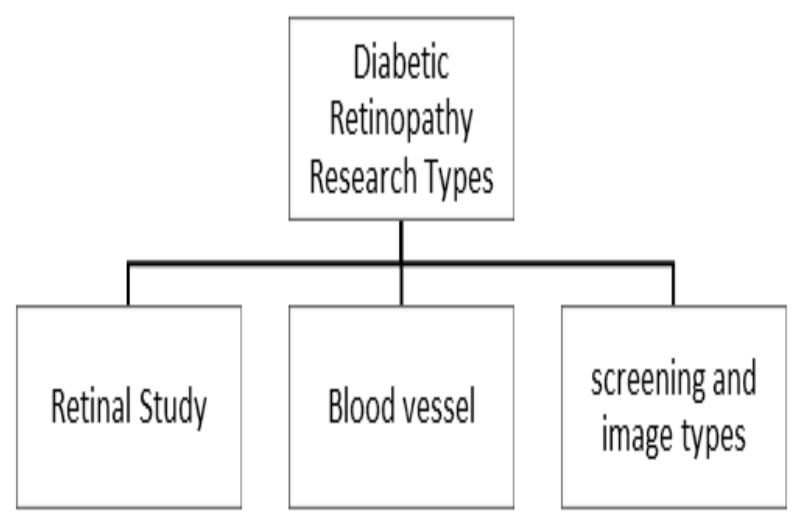

Fig. 2. DR Research Types

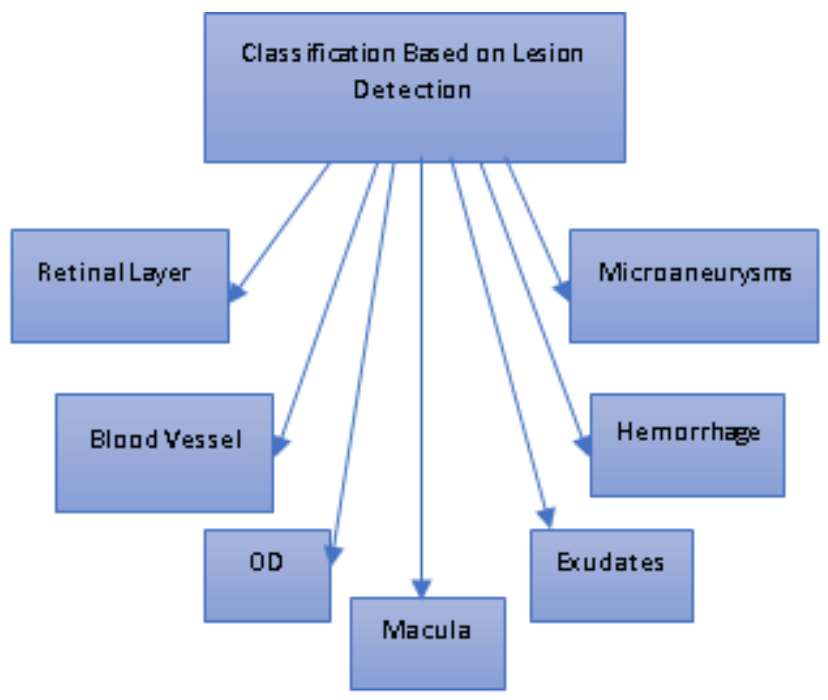

Fig. 3. Classification of DR detection systems by its lesion detection type

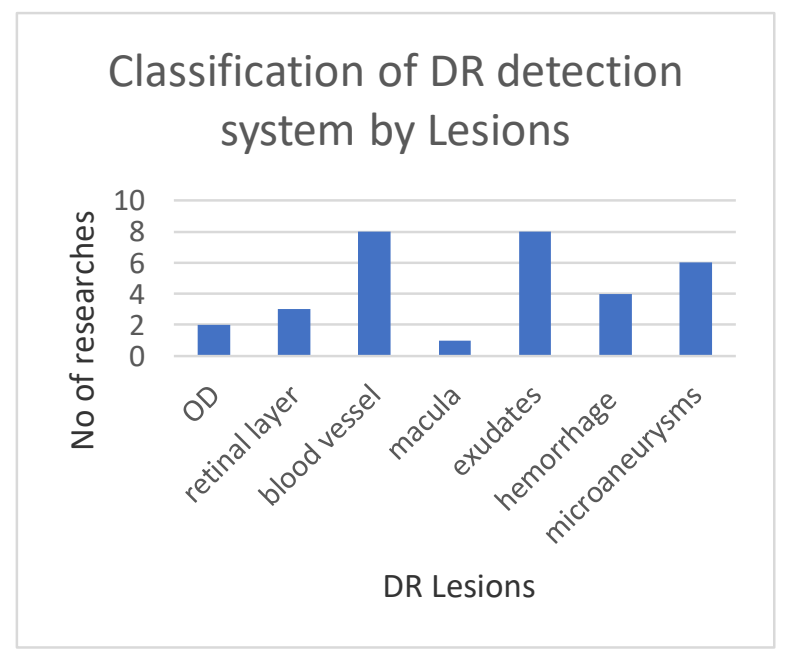

Fig. 4. Classification of DR detection Systems based on Lesions used for the Detection

\section{Datasets}

Many public databases are available online. Few researches have used local primary datasets. Table 1 shows Review on Public DR Dataset available for the study. Many authors have used their own datasets. We observed that for the evaluation of the DR detection systems standard datasets are rarely used. Hence, in future researches algorithms can be tested on multiple datasets to get the efficient detection systems with accurate results.

\section{LITERATURE REVIEW}

\section{A. Feature Extraction Techniques:}

J. Guoa et al. proposed a technique which has inner retina. extraction from the original image depends on segmented layer [1]. 
Morphological operators and thresholding are used for feature extraction in [2], [3], [5], [14], [16], [6]. M. Paranjpe et al. have done texture feature extraction using Gray Level Co-occurrence Matrix (GLCM) and blood vessel was extracted by Morphological Operations [26].

K-means clustering is used by [4] and [8]. Ketki S. Argade et al. have used Sobel edge extraction technique to extract lesions [15]. S. Kaur et al. have used PCA (Principle Component Analysis) for feature extraction and blood vessel segmentation, extracted features are optimized by Lion Optimization Algorithm [18].

Following are the Feature Extraction techniques used by the researchers in DR detection systems,

1) Morphological Operations

2) Gray Level Co-occurrence Matrix (GLCM)

3) Sobel edge extraction technique

4) Fuzzy C Means based
M. Habib et al. have proposed a microaneurysms detection by using ensemble classifier. In the methodology they did MA candidate detection where 70 features were extracted. Fleming's features, shape features \& Moment Invariants, Gaussian features, Intensity features and Morphological features [11]. V. Kurilova et al. have used combination of morphological operations for blood vessel extraction.

These are White top-hat transform (WTH) to extract bright artefacts and black top-hat transform (BTH) to extract dark artefacts.

$$
\begin{aligned}
& \text { WTH }=X-\left(\mathrm{X}^{\mathbb{0}} \mathrm{B}\right) \\
& B T H=(X \cdot B)-X
\end{aligned}
$$

WTH as a subtraction using structural element B and BTH as a subtraction of input image $\mathrm{X}$ [27].

\begin{tabular}{|c|c|c|}
\hline SrNo & Dataset Name & Description \\
\hline 1 & $\begin{array}{l}\text { ROC dataset } \\
\text { (Retinopathy Online Challenge) }\end{array}$ & $\begin{array}{l}\text { - microaneurysm set } \\
\text { - } 100 \text { images: } 50 \text { training images and } 50 \text { testing images. }\end{array}$ \\
\hline 2 & $\begin{array}{l}\text { DIARETDB1 v2 } \\
\text { DIARETDB0 }\end{array}$ & $\begin{array}{l}\text { - } \text { microaneurysm set } \\
\text { - DIARETDB1 v2.1 - } 89 \text { images } \\
\text { - DIARETDB0 -130 images }\end{array}$ \\
\hline 3 & $\begin{array}{l}\text { MESSIDOR database1 } \\
\text { (Methods to Evaluate Segmentation and Indexing Techniques in } \\
\text { the field of Retinal Ophthalmology) }\end{array}$ & $\begin{array}{l}\text { - pupil dilated } 800 \text { images and } 400 \text { images without dilation } \\
\text { - French dataset }\end{array}$ \\
\hline 4 & $\begin{array}{l}\text { DRIVE } \\
\text { (Digital Retinal Images for Vessel Extraction) }\end{array}$ & $\begin{array}{l}\text { - } 400 \text { images of diabetic patients of age group 25-90 years } \\
\text { - Dataset from Netherland } \\
\text { - JPEG compressed images } \\
\text { - Designed for blood vessels segmentation. }\end{array}$ \\
\hline 5 & $\begin{array}{l}\text { STARE } \\
\text { (STructured Analysis of the REtina) }\end{array}$ & $\begin{array}{l}\text { - } 400 \text { images } \\
\text { - Netherland database } \\
\text { - Developed for blood vessel segmentation. }\end{array}$ \\
\hline 6 & $\begin{array}{l}\text { ARIA online } \\
\text { (Automatic Retinal Image Analysis) }\end{array}$ & $\begin{array}{l}\text { - } 143 \text { color fundus images } \\
\text { - UK dataset } \\
\text { - Designed for vessel detection. } \\
\text { - Three image classes: } \\
\text { 1) Age-related macular degeneration (AMD)-23 } \\
\text { 2) Healthy control-group-61 } \\
\text { 3) Diabetic-59 }\end{array}$ \\
\hline 7 & $\begin{array}{l}\text { HRF } \\
\text { (High-Resolution Fundus Image Database) }\end{array}$ & $\begin{array}{l}\text { - Healthy images-15, DR images-15 and glaucomatous images-15 } \\
\text { - Binary gold standard for each image } \\
\text { - For vessel segmentation }\end{array}$ \\
\hline 8 & VICAVR & $\begin{array}{l}\text { - } 58 \text { images } \\
\text { - Used in A/V Ratio computation } \\
\text { - Maintained by VARIA group }\end{array}$ \\
\hline 9 & $\begin{array}{l}\text { CHASE, CHASE_DB1 } \\
\text { (Child Heart and Health Study in England) }\end{array}$ & $\begin{array}{l}\text { - } 28 \text { images of } 14 \text { children } \\
\text { - } \text { retinal images of multiethnic children from England }\end{array}$ \\
\hline
\end{tabular}

TABLE- I: Review on Public DR Datasets 


\begin{tabular}{|c|c|c|}
\hline 10 & $\begin{array}{l}\text { IDRiD } \\
\text { (Indian Diabetic Retinopathy Image Dataset) }\end{array}$ & $\begin{array}{l}\text { - } 516 \text { images } \\
\text { - Designed for Diabetic Retinopathy (DR) and Diabetic Macular Edema } \\
\text { (DME) } \\
\text { - IEEE databases } \\
\text { - created at Eye Clinic, Nanded, (M.S.), India }\end{array}$ \\
\hline 11 & $\begin{array}{l}\text { DR HAGIS database } \\
\text { (Diabetic Retinopathy, Hypertension, Age-related macula } \\
\text { degeneration and Glaucoma ImageS) }\end{array}$ & $\begin{array}{l}\text { - } 39 \text { high-resolution color fundus images } \\
\text { - UK database } \\
\text { - Designed for vessel extraction }\end{array}$ \\
\hline 12 & $\begin{array}{l}\text { FIRE } \\
\text { (Fundus Image Registration Dataset) }\end{array}$ & $\begin{array}{l}\text { - } 129 \text { retinal images with } 134 \text { image pairs } \\
\text { - Greece dataset }\end{array}$ \\
\hline
\end{tabular}

Table- II: Different Segmentation techniques in DR detection systems

\begin{tabular}{|c|c|c|c|}
\hline Sr.no. & Author & Segmentation Technique & Segmentation Accuracy \\
\hline 1 & J. Guoa et al. & $\begin{array}{l}\text { Bayesian posterior probability } \\
\text { Graph Search-Graph Cut } \\
\text { threshold-based }\end{array}$ & Not Available \\
\hline 2 & P. Anupama et al. & $\begin{array}{l}\text { Blood vessel segmentation } \\
\text { using eigen values }\end{array}$ & $94 \%$ \\
\hline 3 & T. Walter et al. & $\begin{array}{l}\text { Morphological filtering technique } \\
\text { watershed transformation }\end{array}$ & $92.40 \%$ \\
\hline 4 & A. Merjora et al. & $\begin{array}{l}\text { Optic disc location using K means clustering } \\
\text { algorithm }\end{array}$ & Not Available \\
\hline 5 & A. Sopharak et al. & Threshold image using Ostu algorithm & $\begin{array}{l}\text { The sensitivity of the exudate } \\
\text { detection algorithm is } 80 \% \text { and } \\
\text { specificity is } 99.5 \% \text {. }\end{array}$ \\
\hline 6 & R. Tanthuwapathom et al. & $\begin{array}{l}\text { adaptive thresholding process, local } \\
\text { thresholding with green channel }\end{array}$ & $\begin{array}{l}85 \% \text { accuracy of exudate } \\
\text { detection }\end{array}$ \\
\hline 7 & J.David et al. & $\begin{array}{l}\text { Haar wavelet, exudates are detected using } \\
\text { thresholding }\end{array}$ & Not Available \\
\hline 8 & G. Hassan et al. & K-means clustering algorithm & $\begin{array}{l}\text { best accuracy-96.25\% with } \\
\text { average accuracy-95.10\% }\end{array}$ \\
\hline 9 & L. Tang et al. & Splat based segmentation & Not Available \\
\hline 10 & K. Argade et al. & $\begin{array}{l}\text { optic disc and cup detection using Sobel Edge } \\
\text { Extraction and Ellipse fitting }\end{array}$ & Not Available \\
\hline 11 & S. Chowhan et al. & $\begin{array}{l}\text { 1) morphological operation } \\
\text { 2) dilation residue edge detector and erosion } \\
\text { residue edge detector }\end{array}$ & Not Available \\
\hline 12 & R. Afrin et al. & Morphological opening technique & Not Available \\
\hline 13 & S. Kaur et al. & edge detection using canny edge operator & $96.17 \%$ \\
\hline 14 & M. Rehman et al. & Discrete Wavelet Transform (DWT) & Not Available \\
\hline 15 & B. Thakar et al. & OD elimination & $\begin{array}{l}\text { 95\% accuracy for optic disc } \\
\text { elimination and 56\% accuracy } \\
\text { for microaneurysms detection. }\end{array}$ \\
\hline
\end{tabular}

\section{B. Classification and Segmentation Techniques:}

It is found that Diabetic Retinopathy can be classified in following stages:

1) Normal DR

2) Mild Non- Proliferative DR

3) Moderate Non- Proliferative DR

4) Severe Non- Proliferative DR

5) Proliferative DR
Table II shows the different segmentation techniques found in DR detection systems with their accuracy in existing literature. 
Table III shows the Literature Review which shows different classification techniques used by different DR detection systems with their accuracy. It also describes the no of classification stages of DR classifiers. M. Rehman et al. [19] uses KNN and SVM classifiers which gives highest DR classification accuracy $98.16 \%$ and $97.85 \%$ respectively.

Table II describes the segmentation techniques used by different researchers in last decade. S. Kaur et al. has proposed a segmentation of DR using edge detection with canny edge operator with $96.17 \%$ accuracy [18]. G. Hassan et al. got higher accuracy by using K-means Clustering algorithm with 95.10\% accuracy [8]. A. Merjora et al. [4] and B. Thakar et al. [20] both used OD elimination for segmentation. Morphological operators are mostly used by researcher for segmentation in DR detection systems [3], [16], [17]. Learning Vector Quantization (LVQ) network [7], back propagation network [7], [14] and deep learning-based CNN classifier [12] are used by researchers for multi-stage Diabetic Retinopathy detection and classification.

Table- III: DR detection systems classification techniques with accuracy of the system

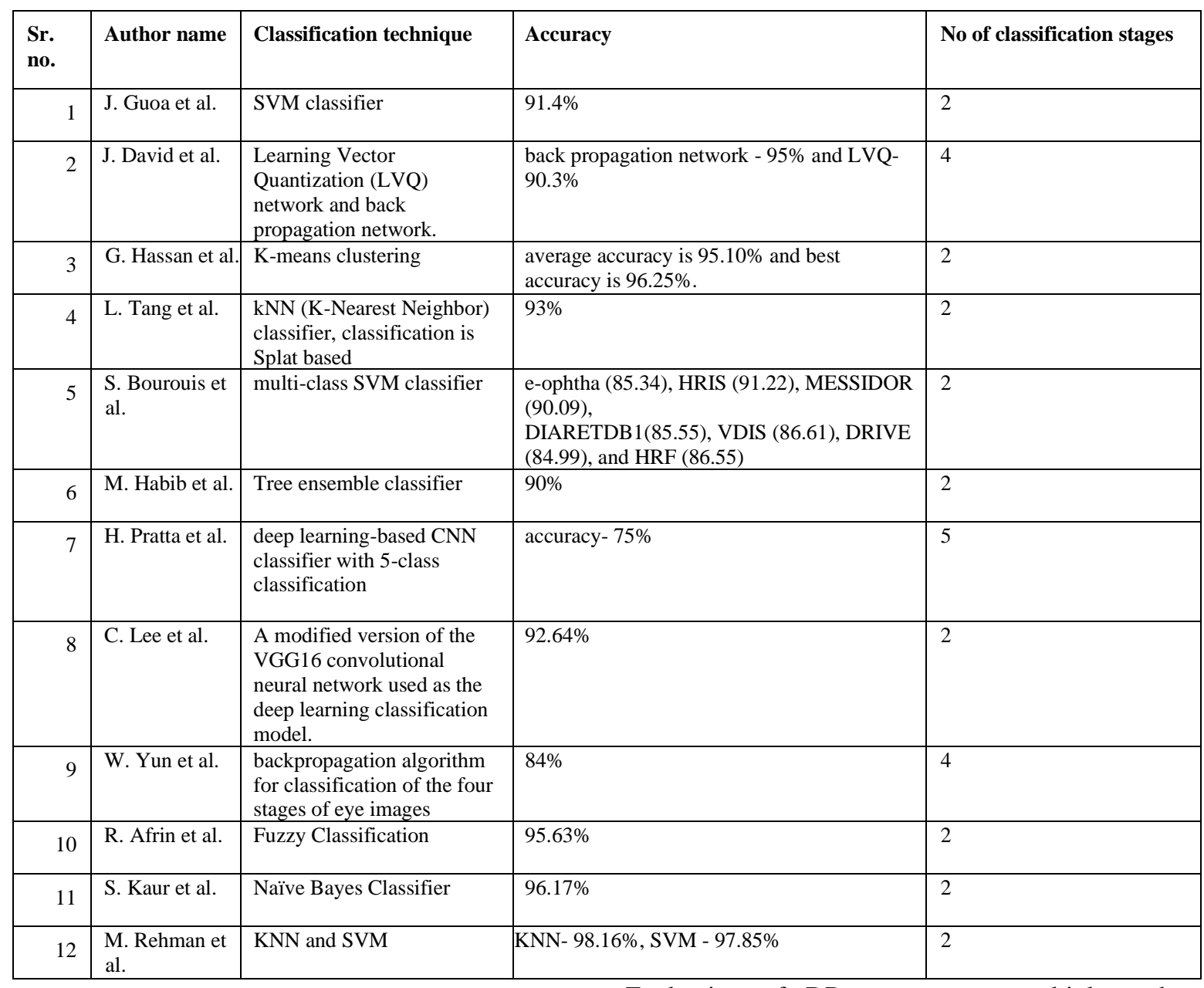

\section{RESEARCH GAP}

With reference to literature review, classification of multi stage DR detection systems is a need for early detection of DR. Existing detection techniques needs to be tested on multiple types of standard databases in terms of fundus, blood vessels, retina. Authors proposed multiple segmentation techniques but no work on multi- lesion segmentation techniques for retina images has been proposed. Child diabetic retinopathy needs to be studied.

\section{CONCLUSION AND FUTURE SCOPE}

This paper gives the review on public datasets, feature extraction techniques and classification techniques used in current Diabetic Retinopathy detection systems. Type of lesions which are the early symptoms of Diabetic Retinopathy are described. This paper gives the overall view on current researches and also highlights the research gaps.
Evaluation of DR systems on multiple and standard databases is done by very few researchers. Further research can be done on DR detection system mainly focus on blood vessel segmentation. Segmentation of all lesions DR detection system is also a challenge for future work. After literature review, we observe that, classification of Diabetic Retinopathy in multiple stages depending upon severity of the disease is also an important area which is less focused.

\section{REFERENCES}

1. J. Guoa, F. Shia, W. Zhua, H. Chenb, and X. Chena, "A Framework for Classification and Segmentation of Branch Retinal Artery Occlusion in SD-OCT," Proc. of SPIE Vol. 9784 978425-2 (spiedigitallibrary.org), 2016. 
2. Anupama. P, Dr S. Nandyal, "Blood Vessel Segmentation using Hessian Matrix for Diabetic Retinopathy Detection,” IEEE, 2017.

3. T. Walter, J. Klein, P. Massin, and A. Erginay, "A Contribution of Image Processing to the Diagnosis of Diabetic Retinopathy - Detection of Exudates in Color Fundus Images of the Human Retina,” IEEE, 2002.

4. A. Merjora.A, Daphny Jass. J.C, Irene Grace.A and J. Mary Subaja, "Detecting the Optical Disc in Digital Fundus Image with Automated and Efficient Method," IEEE - International Conference on Communication and Signal Processing, April 4-6, 2019, India.

5. A. Sopharak, B. Uyyanonvara, S. Barmanb, and T. H. Williamson, "Automatic detection of diabetic retinopathy exudates from non-dilated retinal images using mathematical morphology methods," Computerized Medical Imaging and Graphics, 2008.

6. R. Tanthuwapathom and N. Hnoohom, "Detection of Diabetic Retinopathy Using Image Processing," Advances in Intelligent Systems and Computing 684, Springer International Publishing AG 2018.

7. J. David, R. Krishnan, and S. Kumar. A, "Neural Network Based Retinal Image Analysis," IEEE - Congress on Image and Signal Processing, 2008.

8. G. Hassan, N. El-Bendary, A. Ella Hassanien, A. Fahmy, A. M. Shoeb, and V. Snasel, "Retinal blood vessel segmentation approach based on mathematical morphology," Procedia Computer Science, Elsevier, 2015 .

9. L.Tang, M. Niemeijer, J. M. Reinhardt, M. K. Garvin, and M. Abramoff, "Splat Feature Classification with Application to Retinal Hemorrhage Detection in Fundus Images," IEEE Transactions on Medical Imaging, Vol. 32, 2013.

10. S. Bourouis, A. Zaguia, N. Bouguila and R. Alroobaea, "Deriving Probabilistic SVM Kernels from Flexible Statistical Mixture Models and Its Application to Retinal Images Classification,” IEEE, 2019.

11. M.M. Habib, R.A. Welikala, A. Hoppe, C.G. Owen, A.R. Rudnicka, and S.A. Barman, "Detection of microaneurysms in retinal images using an ensemble classifier," Elsevier, Informatics in Medicine Unlocked, 2017.

12. H. Pratta, F. Coenen, D. M Broadbent, S. P Harding, and Y. Zheng, "Convolutional Neural Networks for Diabetic Retinopathy," Procedia Computer Science, Elsevier (International Conference on Medical Imaging Understanding and Analysis 2016), 2016.

13. C. S. Lee, D. M. Baughman, and A. Y. Lee, "Deep Learning Is Effective for Classifying Normal versus Age-Related Macular Degeneration OCT Images," American Academy of Ophthalmology, Elsevier, 2016.

14. W. Li Yun, U. R. Acharya, Y.V. Venkatesh, C. Chee, L. Choo Min, and E.Y.K. Ng, "Identification of different stages of diabetic retinopathy using retinal optical images," Information Sciences, Elsevier, 2008.

15. K. S. Argade, K. A. Deshmukh, M. M. Narkhede, N. N. Sonawane, and S. Jore," Automatic Detection of Diabetic Retinopathy using Image Processing and Data Mining Techniques," International Conference on Green Computing and Internet of Things (ICGCloT), IEEE, 2015.

16. S. S. Chowhan, R.S. Deore, and S. A. Naik, "Retinal Vessel Segmentation of Non-Proliferative Diabetic Retinopathy,' International Journal of Applied Evolutionary Computation, 2019.

17. R. Afrin, and P. Chandra Shill, "Automatic Lesions Detection and Classification of Diabetic Retinopathy Using Fuzzy Logic,' International Conference on Robotics, Electrical and Signal Processing Techniques (ICREST), IEEE, 2019.

18. S. Kaur, and K. Singh Mann, "Optimized Technique for Detection of Diabetic Retinopathy using Segmented Retinal Blood Vessels," International Conference on Automation, Computational and Technology Management (ICACTM), Amity University, IEEE, 2019.

19. M. ur Rehman, Z. Abbas, S. Haris Khan, S. Hikmat Ghani, and N.,' Diabetic Retinopathy Fundus Image Classification using Discrete Wavelet Transform," IEEE, 2018.

20. B. Thakar, S. Patel, V. Palod, A. Shetty, P. Hatode, and J. Khanapuri, "Automatic Detection of Microaneurysms in Diabetic Retinopathy using Python," 2nd International Conference on Advances in Science \& Technology, 2019.

21. A. Guptaa, and R. Chhikarab," Diabetic Retinopathy: Present and Past," International Conference on Computational Intelligence and Data Science, Procedia Computer Science 132 (2018) 1432-1440, Elsevier, 2018.

22. M.M. Fraz, P. Remagnino, A. Hoppe, B. Uyyanonvara, A.R Rudnicka, C.G. Owenc, and S.A. Barmana, "Blood vessel segmentation methodologies in retinal images- A survey," computer methods and programs in biomedicine 108 (2012) 407-433, Elsevier, 2012.

23. A. Hajdu, T. Peto, Z. Torok, and A. Biro, "Extracting metadata from fundus images for the screening of diabetic retinopathy," Conference
Paper, DOI: 10.1109/WISP.2009.5286555 - Source: IEEE Xplore, 2009.

24. R.J. Windera, P.J. Morrow, I.N. McRitchie, J.R. Bailie and P.M. Hart, "Algorithms for digital image processing in diabetic retinopathy," Computerized Medical Imaging and Graphics 33 (2009) 608-622, Elsevier, 2009.

25. K. Noronha and K. Prabhakar Nayak, "Fundus Image Analysis for the Detection of Diabetic Eye Diseases-A Review," International Conference on Biomedical Engineering (ICoBE), Penang, Malaysia, IEEE, February 2012.

26. M. J. Paranjpe \& M. N. Kakatkar, (2013). "Automated Diabetic Retinopathy severity classification using Support Vector Machine", IJRSAT, Vol 3, Pg-086-091, May-June 2013.

27. V. Kurilova, J. Pavlovicova, M. Oravec, R. Rakar, I. Marcek, "Retinal Blood Vessels Extraction Using Morphological Operations", IEEE, 2015.

28. https://eagleeyecentre.com.sg.

\section{AUTHORS PROFILE}

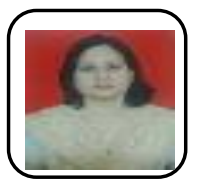

Jyoti P. Sawant is a post graduate in Computer Science from Swami Ramanand Teerth Marathwada University, Nanded and pursuing her Ph.D. in Faculty of Computer Studies, Symbiosis International University, Pune, Maharashtra, India. She has experience of teaching in Computer Science and Information Technology for undergraduate and post-graduate students.

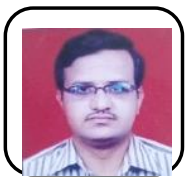

Sachin A. Naik is a post graduate in Computer Science from Dr. Babasaheb Ambedkar Marathwada University, Aurangabad and completed his doctoral degree from Symbiosis International (Deemed University). Currently he is working as Assistant Professor in Symbiosis Institute of Computer Studies and Research, a constituent of Symbiosis International (Deemed University). He has a strong teaching experience in the field of computer science and information technology. His area of expertise is in digital image processing, pattern recognition, software engineering and object-oriented programming. He has published papers in referred/indexed journals including SCOPUS and conferences of international repute.

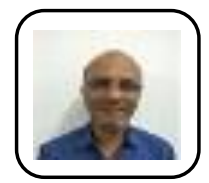

Santosh Chowhan is a postgraduate in computer science from Dr. Babasaheb Ambedkar Marathwada University, Aurangabad. He completed his doctoral degree from SRTM University Nanded in the field of biometric image processing. Currently he is working as Assistant Professor in Symbiosis Institute of Computer Studies and Research, a constituent of Symbiosis International (Deemed University). He has a strong teaching experience in the field of computer science and information technology.

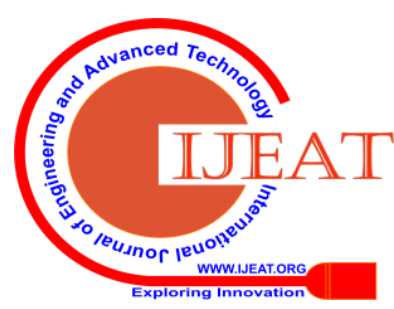

